\title{
Serum Superoxide Dismutase Is Associated with Vascular Structure and Function in Hypertensive and Diabetic Patients
}

\author{
Manuel A. Gómez-Marcos, ${ }^{1}$ Ana M. Blázquez-Medela, ${ }^{2}$ Luis Gamella-Pozuelo, ${ }^{2}$ \\ José I. Recio-Rodriguez, ${ }^{1}$ Luis García-Ortiz, ${ }^{1}$ and Carlos Martínez-Salgado ${ }^{2,3}$ \\ ${ }^{1}$ Research Unit, Primary Care Centre La Alamedilla, SACYL, Avenida de los Comuneros 27, 37003 Salamanca, Spain \\ ${ }^{2}$ Institute of Biomedical Research of Salamanca (IBSAL), Renal and Cardiovascular Pathophysiology Unit, \\ Department of Physiology and Pharmacology, University of Salamanca, Avenida Campo Charro s $/ n, 37007$ Salamanca, Spain \\ ${ }^{3}$ Institute of Studies in Health Sciences of Castilla y León (IECSCYL), Research Unit, University Hospital of Salamanca, \\ Paseo San Vicente 58-182, 37007 Salamanca, Spain
}

Correspondence should be addressed to Carlos Martínez-Salgado; carlosms@usal.es

Received 17 February 2015; Revised 9 June 2015; Accepted 14 June 2015

Academic Editor: Matías Mosqueira

Copyright ( 2016 Manuel A. Gómez-Marcos et al. This is an open access article distributed under the Creative Commons Attribution License, which permits unrestricted use, distribution, and reproduction in any medium, provided the original work is properly cited.

\begin{abstract}
Oxidative stress is associated with cardiac and vascular defects leading to hypertension and atherosclerosis, being superoxide dismutase (SOD) one of the main intracellular antioxidant defence mechanisms. Although several parameters of vascular function and structure have a predictive value for cardiovascular morbidity-mortality in hypertensive patients, there are no studies on the involvement of SOD serum levels with these vascular parameters. Thus, we assessed if SOD serum levels are correlated with parameters of vascular function and structure and with cardiovascular risk in hypertensive and type 2 diabetic patients. We enrolled 255 consecutive hypertensive and diabetic patients and 52 nondiabetic and nonhypertensive controls. SOD levels were measured with an enzyme-linked immunosorbent assay kit. Vascular function and structure were evaluated by pulse wave velocity, augmentation index, ambulatory arterial stiffness index, and carotid intima-media thickness. We detected negative correlations between SOD and pressure wave velocity, peripheral and central augmentation index and ambulatory arterial stiffness index, pulse pressure, and plasma HDL-cholesterol, as well as positive correlations between SOD and plasma uric acid and triglycerides. Our study shows that SOD is a marker of cardiovascular alterations in hypertensive and diabetic patients, since changes in its serum levels are correlated with alterations in vascular structure and function.
\end{abstract}

\section{Introduction}

Hypertension is quantitatively the most important risk factor for premature cardiovascular disease; essential hypertension and diabetes are characterized by endothelial dysfunction mediated by an impaired $\mathrm{NO}$ availability secondary to oxidative stress production [1]. Vascular disease is one of the main causes for disability and death in patients with diabetes mellitus [2], which invariably show endothelial dysfunction as well as associated cardiovascular risk factors as hypertension, obesity, and dyslipidemia [3]. Either dyslipidemia, hyperinsulinemia, insulin resistance, or hyperglycemia contributes to the development of endothelial dysfunction [4].
Arterial stiffness, estimated by pulse wave velocity (PWV) determination, has an independent predictive value for cardiovascular events [5], is associated with the severity of coronary artery disease, and is impaired in coronary atherosclerosis [6]. The ambulatory arterial stiffness index (AASI) is related to cardiovascular morbidity-mortality [7] and to the associated target organ damage in hypertensive patients [8]. AASI is very useful for assessing arterial stiffness and is an independent predictor of cardiovascular mortality and morbidity in patients with cardiovascular disease and in healthy individuals. We have previously shown that AASI is positively correlated with carotid intima-media thickness (IMT) and PWV and negatively correlated with glomerular filtration [9]. Another parameter to measure wave reflection 
and arterial stiffness is the augmentation index (AIx), which is a predictor of adverse cardiovascular events, and higher values are associated with target organ damage [10].

The role of reactive oxygen species (ROS) in the pathophysiology of cardiovascular diseases has been described, as oxidative stress is associated with cardiac and vascular defects leading to hypertension and atherosclerosis [11], but direct cause and effect relationships have not been clearly defined. Although ROS originate from different sources, the vascular NADPH seems to be one of the main sources in cardiovascular pathophysiology [12]. Elevated levels of superoxide anion have been detected in essential hypertension [13] and in the development of atherosclerosis [14]. The enzyme superoxide dismutase (SOD) is an intracellular antioxidant defence mechanism which catalyses the dismutation of superoxide radical into $\mathrm{H}_{2} \mathrm{O}_{2}$ and oxygen [11]. SOD has a protective role in atherogenesis [15] and improves hypertension modulating vasodilation, vasoconstriction, vascular remodelling, and cardiac hypertrophy, playing a relevant role in the development and the maintenance of chronic hypertension in various organs [16].

However, so far there have been no studies that evaluate the possible relationship between serum levels of this enzyme and different vascular parameters with a predictive value on cardiovascular risk. Thus, we have assessed the relationship between SOD serum levels and parameters of vascular function and structure (PWV, AASI, IMT, and AIx) as well as cardiovascular risk in hypertensive and type 2 diabetic patients.

\section{Materials and Methods}

This is a cross-sectional study performed in 307 consecutive patients (54 with type 2 diabetes and hypertension, 16 nonhypertensive diabetic, 185 hypertensive nondiabetic patients, and 52 nondiabetic and nonhypertensive controls), enrolled in the study over a period of 24 months (from January 2008 to January 2010) in the Primary Care Research Unit of La Alamedilla Health Centre (Castilla y León Health Service-SACYL), Salamanca, Spain, which complied with the inclusion/exclusion criteria.

Inclusion Criteria are as follows: patients aged 2080 years, diagnosed with type 2 diabetes mellitus and/or hypertension. Exclusion criteria are as follows: patients with secondary hypertension, patients unable to comply with the protocol requirements (psychological and/or cognitive disorders, failure to cooperate, educational limitations and problems in understanding written language, and failure to sign the informed consent document), patients participating or who were going to participate in clinical trials during the study, and patients with serious comorbidities representing a threat to life (known coronary or cerebrovascular atherosclerotic disease, heart failure, moderate or severe chronic obstructive pulmonary disease, walkinglimiting musculoskeletal disease, advanced respiratory, renal or hepatic disease, severe mental diseases, treated oncological disease diagnosed in the past 5 years, pregnant women, and terminal patients). Most of the patients with hypertension and diabetes received drug therapy (except those controlled by diet), which is described in Table 1 .

Sample size calculation indicated that the 307 patients included in the study were sufficient to detect a coefficient correlation of 0.16 between superoxide dismutase with parameters of vascular function and structure in a two-sided test with an alpha risk of 0.05 and a power of $80 \%$.

Hypertension was diagnosed as recommended by The Task Force for the Management of Arterial Hypertension of the European Society of Hypertension and of the European Society of Cardiology [17]. Diabetes was diagnosed as recommended by the Expert Committee on the Diagnosis and Classification of Diabetes Mellitus [18].

2.1. Ethical and Legal Issues. The experimental protocol was in accordance with the Declaration of Helsinki (2000) of the World Medical Association and approved by the Ethics Committee of the University Hospital of Salamanca (Spain) and complied with Spanish data protection law 15/1999 and its developed specifications (RD 1720/2007). Each patient signed a participation informed consent after full explanation of the study.

2.2. Sociodemographic and Cardiovascular Variables. We evaluated patient age and sex, hypertension, dyslipidemia, alcohol consumption, smoking, history of premature cardiovascular disease (before 55 years of age in males and before 65 in females), and patients on treatment with antihypertensive, antidiabetic, lipid lowering, and antiaggregant drugs.

2.3. Serum SOD and 8-Hydroxy-2-deoxyguanosine Determination. Serum concentrations of $\mathrm{Cu} / \mathrm{Zn} \mathrm{SOD}$ and 8hydroxy-2-deoxyguanosine were determined with ELISA kits (Cu/Zn SOD: Northwest Life Sciences Inc., Vancouver, WA, USA; 8-hydroxy-2-deoxyguanosine: Abcam, Cambridge, UK), according to the instructions of the manufacturer. Absorbance was read on a spectrophotometer (Thermo Luminoskan Ascent, Waltham MA, USA) at $450 \mathrm{~nm}(\mathrm{Cu} / \mathrm{Zn}$ SOD) and 410 (8-hydroxy-2-deoxyguanosine).

2.4. Other Biochemical Determinations. Blood samples were collected after patient fasting for at least 8 hours. Determinations are as follows: creatinine, basal glucose, HbAlc, uric acid, HDL-cholesterol, LDL-cholesterol, total cholesterol, and triglycerides. The parameters were measured on a blind basis in a General Hospital Biochemistry laboratory using standard automatized techniques.

2.5. Blood Pressure Determination. Office blood pressure evaluation involved three systolic (SBP) and diastolic blood pressure (DBP) measurements, using the average of the last two measurements, with a validated OMRON model M10IT sphygmomanometer (Omron Health Care, Kyoto, Japan), following the recommendations of the European Society of Hypertension [19]. Pulse pressure was estimated with the mean values of the second and third measurements.

Ambulatory blood pressure monitoring (ABPM) was performed on a day of standard activity using a control 
TABLE 1: Characteristics of study population.

\begin{tabular}{|c|c|c|c|c|c|c|}
\hline & All patients & DIA + HYP & DIA & HYP & Control & $p$ \\
\hline Number & 307 & 54 & 16 & 185 & 52 & \\
\hline Age (years) & $54.76 \pm 11.69$ & $60.75 \pm 8.35$ & $55.27 \pm 12.82$ & $55.26 \pm 10.99$ & $46.63 \pm 12.54$ & $<0.001$ \\
\hline Male sex $(N, \%)$ & $191(62.21 \%)$ & $39(72.22 \%)$ & $11(68.75 \%)$ & $108(58.38 \%)$ & $33(63.46 \%)$ & 0.286 \\
\hline Superoxide dismutase (ng/mL) & $123.48 \pm 57.20$ & $134.76 \pm 63.60$ & $112.00 \pm 35.23$ & $117.63 \pm 47.69$ & $137.91 \pm 82.68$ & 0.079 \\
\hline 8-Hydroxy-2-deoxyguanosine (ng/mL) & $6.97 \pm 5.07$ & $7.97 \pm 5.52$ & $6.24 \pm 4.17$ & $6.35 \pm 5.13$ & $7.40 \pm 4.76$ & 0.377 \\
\hline Systolic blood pressure (mmHg) & $138.26 \pm 17.15$ & $142.59 \pm 18.20$ & $119.31 \pm 9.61$ & $143.66 \pm 16.09$ & $126.26 \pm 8.92$ & $<0.001$ \\
\hline Diastolic blood pressure (mmHg) & $86.89 \pm 10.92$ & $84.94 \pm 12.07$ & $74.13 \pm 5.19$ & $90.62 \pm 9.88$ & $79.56 \pm 6.66$ & $<0.001$ \\
\hline Pulse pressure $(\mathrm{mmHg})$ & $52.49 \pm 12.37$ & $57.37 \pm 12.92$ & $45.39 \pm 10.15$ & $53.16 \pm 12.23$ & $46.91 \pm 10.00$ & $<0.001$ \\
\hline IMT medium average (mm) & $0.73 \pm 0.11$ & $0.80 \pm 0.11$ & $0.73 \pm 0.12$ & $0.73 \pm 0.10$ & $0.68 \pm 0.14$ & $<0.001$ \\
\hline IMT maximum average (mm) & $0.90 \pm 0.14$ & $0.98 \pm 0.12$ & $0.90 \pm 0.15$ & $0.90 \pm 0.12$ & $0.84 \pm 0.17$ & $<0.001$ \\
\hline Pulse wave velocity (m/s) & $8.98 \pm 2.22$ & $10.39 \pm 2.35$ & $8.65 \pm 2.27$ & $9.00 \pm 2.09$ & $7.59 \pm 1.61$ & $<0.001$ \\
\hline Peripheral augmentation index & $92.76 \pm 21.43$ & $95.85 \pm 20.87$ & $88.94 \pm 22.34$ & $95.15 \pm 21.45$ & $82.22 \pm 18.63$ & 0.001 \\
\hline Central augmentation index & $30.31 \pm 11.66$ & $31.35 \pm 10.67$ & $30.44 \pm 12.10$ & $31.81 \pm 10.94$ & $23.85 \pm 13.04$ & $<0.001$ \\
\hline AASI & $38.25 \pm 6.05$ & $41.39 \pm 5.96$ & $38.27 \pm 5.44$ & $37.71 \pm 5.89$ & $36.92 \pm 5.96$ & $<0.001$ \\
\hline D’Agostino cardiovascular risk & $19.73 \pm 16.27$ & $36.59 \pm 18.22$ & $17.34 \pm 11.88$ & $18.00 \pm 13.72$ & $8.59 \pm 8.24$ & $<0.001$ \\
\hline Smokers $(N, \%)$ & $73(23.77 \%)$ & $13(24.07 \%)$ & $4(25 \%)$ & $38(20.54 \%)$ & $18(34.62 \%)$ & 0.202 \\
\hline HDL (mg/dL) & $51.98 \pm 12.45$ & $47.48 \pm 9.73$ & $50.75 \pm 12.68$ & $52.86 \pm 12.63$ & $53.96 \pm 13.42$ & 0.023 \\
\hline LDL (mg/dL) & $125.91 \pm 32.76$ & $108.13 \pm 27.96$ & $108.50 \pm 22.07$ & $132.71 \pm 34.03$ & $125.75 \pm 26.68$ & $<0.001$ \\
\hline Total cholesterol (mg/dL) & $204.40 \pm 36.98$ & $187.46 \pm 34.70$ & $183.81 \pm 23.66$ & $211.44 \pm 37.58$ & $203.25 \pm 32.77$ & $<0.001$ \\
\hline Alcohol consumption (units/week) & $10.98 \pm 20.28$ & $14.94 \pm 21.35$ & $3.69 \pm 4.87$ & $9.63 \pm 15.81$ & $13.87 \pm 32.15$ & 0.111 \\
\hline HbAlc (\%) & $5.41 \pm 1.05$ & $6.84 \pm 1.40$ & $6.68 \pm 0.80$ & $5.05 \pm 0.46$ & $4.83 \pm 0.40$ & $<0.001$ \\
\hline Glycemia (mg/dL) & $98.44 \pm 30.44$ & $136.61 \pm 46.27$ & $132.63 \pm 32.63$ & $88.63 \pm 11.14$ & $83.19 \pm 8.75$ & $<0.001$ \\
\hline Antihypertensive drugs $(N, \%)$ & $143(46.58 \%)$ & $48(88.89 \%)$ & $0(0.00 \%)$ & $95(51.35 \%)$ & $0(0.00 \%)$ & $<0.001$ \\
\hline Antidiabetic drugs $(N, \%)$ & $66(21.50 \%)$ & $51(94.44 \%)$ & $15(93.75 \%)$ & $0(0.00 \%)$ & $0(0.00 \%)$ & $<0.001$ \\
\hline Lipid-lowering drugs $(N, \%)$ & $84(27.36 \%)$ & $32(59.26 \%)$ & $6(37.50 \%)$ & $43(23.24 \%)$ & $3(5.77 \%)$ & $<0.001$ \\
\hline Antiaggregants $(N, \%)$ & $61(19.87 \%)$ & $29(53.70 \%)$ & $6(37.50 \%)$ & $23(12.43 \%)$ & $3(5.77 \%)$ & $<0.001$ \\
\hline
\end{tabular}

Demographic, physical, and medical characteristics and drug therapies of patients included in the study. Data are expressed as mean \pm standard deviation or percentage. AASI: ambulatory arterial stiffness index; DIA: diabetic nonhypertensive patients; DIA + HYP: diabetic hypertensive patients; HbAlc: glycosylated haemoglobin; HDL: cholesterol associated with high density lipoproteins; HYP: hypertensive patients; IMT: intima-media thickness; LDL: cholesterol associated with low density lipoproteins; $p$ : $p$ value, statistically significant differences (ANOVA).

system (Spacelabs 90207, Healthcare, Issaquah, Washington, USA). We obtained blood pressure measurements every 20 min (waking period) and every 30 min (resting period). Valid records of readings were $80 \%$ of the total.

2.6. Determination of Pulse Wave Velocity (PWV) and Peripheral (PAIX) and Central (CAIX) Augmentation Index. These parameters were estimated using the SphygmoCor System (AtCor Medical Pty Ltd., Head Office, West Ryde, Australia). Pulse wave analysis was performed with a sensor in the radial artery, using mathematical transformations to estimate the aortic pulse wave. CAIx was estimated from the morphology of the aortic wave using the following formula: increase in central pressure $\times 100 /$ pulse pressure. PAIx was calculated as follows: (second peak SBP [SBP2] - [DBP])/(first peak SBP - DBP) $\times 100(\%)$. Measurements reliability was evaluated using the CAIx intraclass correlation coefficient, which showed values of 0.974 (95\% CI: 0.936-0.989) for intraobserver agreement on repeated measurements in 22 subjects. According to the Bland-Altman analysis, the limit of intraobserver agreement was 0.454 (95\% CI: -9.876-10.785). The carotid-femoral pulse wave was analysed estimating the delay with respect to the ECG wave and calculating the PWV. Distance measurements were taken with a measuring tape from the sternal notch to the carotid and femoral arteries at the sensor location.

2.7. Ambulatory Arterial Stiffness Index (AASI). Arterial stiffness was evaluated with AASI, defined as one minus the regression slope of DBP over SBP readings obtained from individual 24-hour blood pressure recordings. The stiffer the arterial tree, the closer the regression slope and AASI to 0 and 1 , respectively.

2.8. Assessment of Carotid Intimamedia Thickness (C-IMT). Carotid ultrasound to assess carotid IMT was performed by two investigators trained for this purpose before starting the study. The reliability of such recordings was evaluated before the study, using the intraclass correlation coefficient, which showed values of 0.97 ( $95 \%$ CI: 0.94 to 0.99 ) for intraobserver agreement on repeated measurements in 20 subjects, and 0.90 ( $95 \%$ CI: 0.74 to 0.96 ) for interobserver agreement. According to the Bland-Altman analysis, the limit of interobserver agreement was 0.02 (95\% limits of agreement: $-0.05-0.10$ ), 
TABLE 2: Pearson correlations between serum superoxide dismutase and parameters of vascular structure and function and cardiovascular risk.

\begin{tabular}{|c|c|c|c|c|c|}
\hline & \multicolumn{5}{|c|}{ Superoxide dismutase } \\
\hline & All patients & DIA + HYP & DIA & HYP & Control \\
\hline Intima-media thickness medium average & -0.08 & 0.06 & 0.45 & -0.12 & -0.22 \\
\hline Intima-media thickness maximum average & -0.06 & 0.06 & 0.41 & -0.07 & -0.22 \\
\hline Pulse wave velocity & $-0.15^{*}$ & -0.06 & -0.03 & $-0.16^{*}$ & 0.25 \\
\hline Peripheral augmentation index & $-0.16^{* *}$ & -0.08 & -0.09 & -0.13 & $-0.37^{*}$ \\
\hline Central augmentation index & $-0.16^{* *}$ & -0.01 & -0.18 & -0.10 & $-0.35^{*}$ \\
\hline AASI & $-0.19^{* *}$ & $-0.32^{*}$ & 0.20 & $-0.24^{* *}$ & -0.15 \\
\hline Pulse pressure & $-0.17^{* *}$ & -0.20 & -0.07 & $-0.18^{*}$ & -0.19 \\
\hline D’Agostino cardiovascular risk & -0.03 & -0.09 & 0.46 & -0.06 & -0.10 \\
\hline 8-Hydroxy-2-deoxyguanosine & -0.17 & -0.29 & 0.21 & -0.21 & -0.22 \\
\hline Uric acid & $0.19^{* *}$ & 0.19 & 0.25 & $0.20^{* *}$ & 0.28 \\
\hline HDL & $-0.18^{* *}$ & -0.10 & -0.22 & $-0.18^{*}$ & -0.28 \\
\hline LDL & 0.02 & -0.06 & 0.40 & 0.13 & -0.10 \\
\hline Triglycerides & $0.19^{* *}$ & 0.19 & 0.44 & $0.20^{* *}$ & 0.25 \\
\hline
\end{tabular}

AASI: ambulatory arterial stiffness index; DIA: diabetic nonhypertensive patients; DIA + HYP: diabetic hypertensive patients; HDL: cholesterol associated with high density lipoproteins; HYP: hypertensive patients; LDL: cholesterol associated with low density lipoproteins. Statistical significant differences: ${ }^{*} p<0.05$; ${ }^{* *} p<0.01$.

and the limit of intraobserver agreement was $0.01(95 \%$ : $-0.03-0.06$ ). A Sonosite Micromax ultrasound (Sonosite Inc., Bothell, Washington, USA) device paired with a 5$10 \mathrm{MHz}$ multifrequency high-resolution linear transducer with Sonocal software was used for automatic measurements of IMT to optimize reproducibility. Measurements were made of the common carotid after the examination of a longitudinal section of $10 \mathrm{~mm}$ at a distance of $1 \mathrm{~cm}$ from the bifurcation, performing measurements in the proximal wall and in the distal wall in the lateral, anterior, and posterior projections, following an axis perpendicular to the artery to discriminate two lines: one for the intima-blood interface and the other for the media-adventitious interface. 6 measurements were obtained in both the right and the left carotid, using average values (average C-IMT) and maximum values (maximum CIMT) automatically calculated by the software [20]. The average IMT was considered abnormal if it measured $0.90 \mathrm{~mm}$, or if there were atherosclerotic plaques with a diameter of $1.5 \mathrm{~mm}$ or a focal increase of $0.5 \mathrm{~mm}$ or $50 \%$ of the adjacent IMT [17].

2.9. Cardiovascular Risk Assessment. Risk of cardiovascular morbidity and mortality was estimated using the risk equation (D'Agostino scale) based on the Framingham study [21]. The individuals performing the different tests were blinded to the clinical data of the patient. All organ damage assessment measures were made within a period of 10 days.

2.10. Statistical Analysis. Data input was made using the Teleform system (Autonomy Cardiff, Vista, CA, USA), exporting the data to the PASW version 18.0 statistical package (SPSS Inc., Chicago IL, USA). Data was presented as mean \pm standard deviation or percentage. One-way analysis of variance (ANOVA) for independent samples was used to compare quantitative variables among SOD quartiles. Pearson correlation test was used to analyze associations between quantitative variables. Using the general linear model procedure, we have conducted two multivariate analyses in which we have considered AASI, PAIx, PWV, and IMT as dependent variables, and SOD quartiles as independent variables. We have performed a first model without adjustments and a second model adjusted for age in each of the independent variables (represented in Figure 1). Hypothesis contrasting established an alpha risk factor of 0.05 as the limit of statistical significance.

\section{Results}

General and medical characteristics of the patients are presented in Table 1. The average age of the patients was 55 years, and 62 percent of them were male. 143 subjects (46.6\%) have antihypertensive treatment, 66 (21.5\%) with antidiabetic treatment, and 84 (27.4\%) with lipid-lowering therapy, of whom $74(24 \%)$ are taking statins, 7 (2.3\%) are taking fibrates, and 6 (2\%) other lipid-lowering drugs. The values of the different parameters of vascular structure and function (IMT, PWV, PAIx, CAIx, and AASI) are lower in the control group and higher in the group with diabetes and hypertension associated $(p<0.001)$ (Table 1$)$. Plasma levels of 8-hydroxy-2-deoxyguanosine, one of the predominant forms of free radical-induced oxidative lesions, were similar in the different groups of patients.

In our study population, serum SOD was inversely correlated with PWV, PAIx, CAIx, AASI, and pulse pressure. We also found a negative correlation with plasma HDLcholesterol and a positive correlation with uric acid levels and triglycerides only in hypertensive patients, correlations that remain after adjusting the data for the intake of antihypertensive and lipid-lowering drugs (Table 2). 


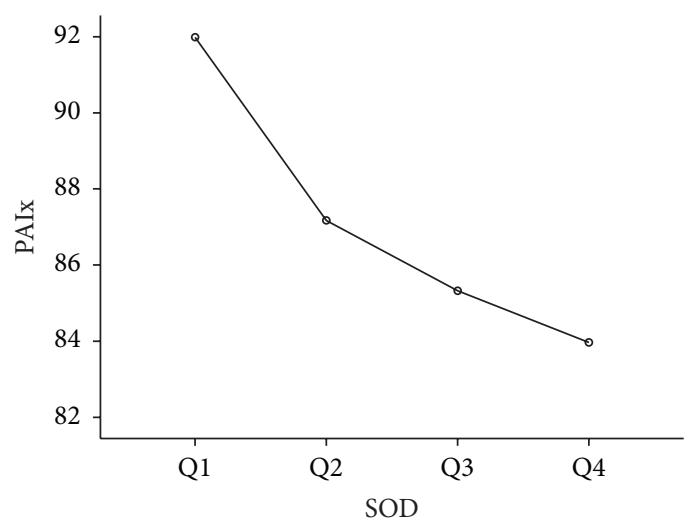

(a)

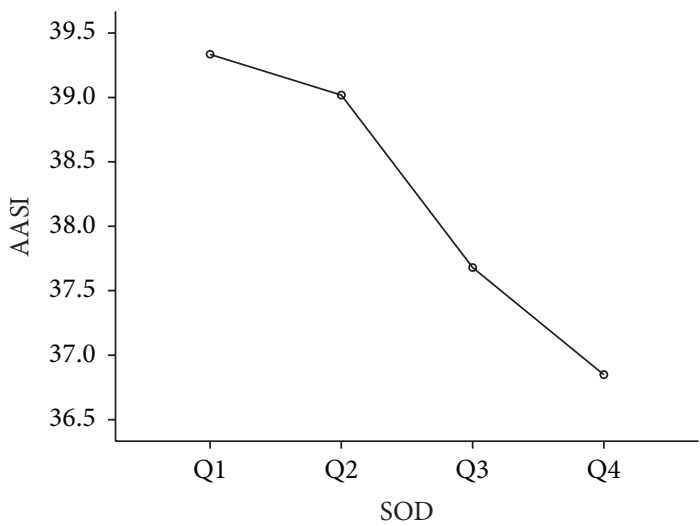

(c)

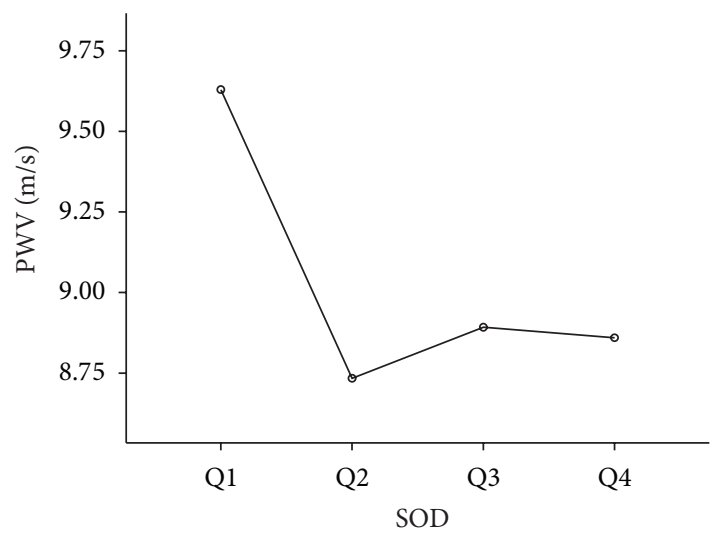

(e)

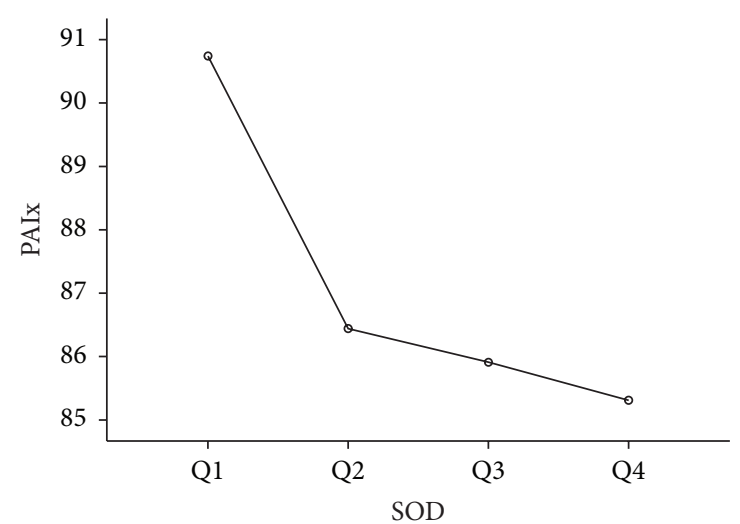

(b)

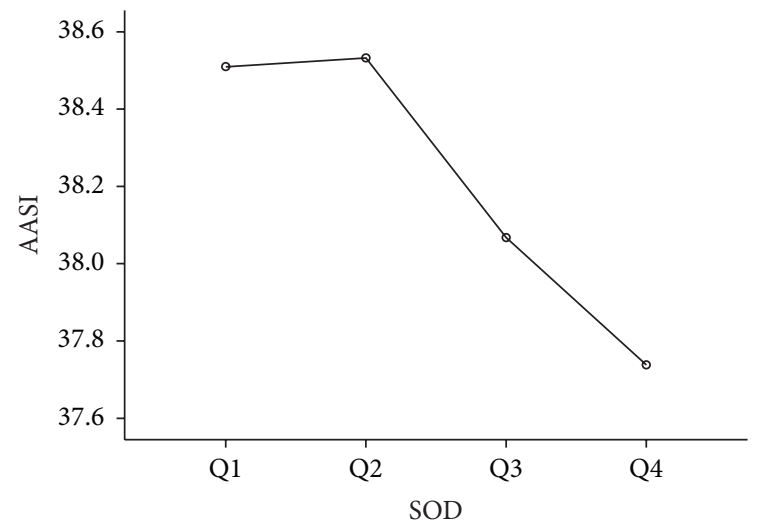

(d)

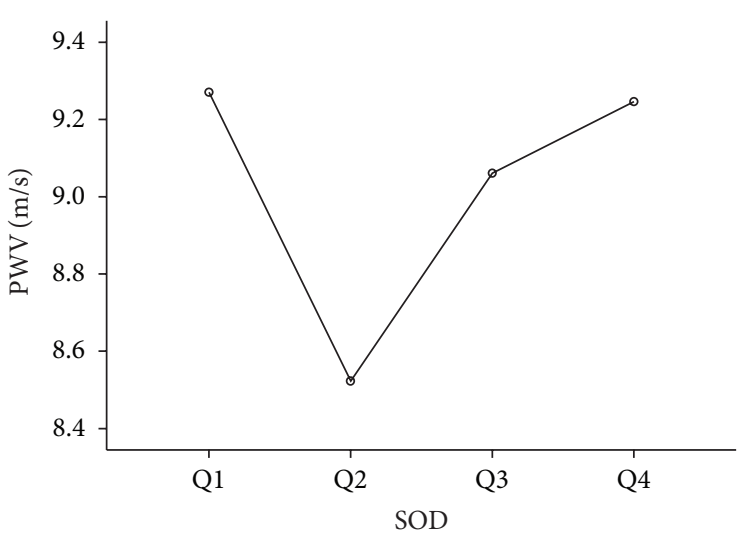

(f)

FIGURE 1: Peripheral augmentation index, ambulatory arterial stiffness index, and pulse wave velocity values divided into 4 quartiles according to SOD serum levels. Multivariate analysis: AASI, PAIx, PWV, and IMT are dependent variables, and SOD is the independent variable. (b), (d), and (f) show values after adjusting for age the independent variable. AASI: ambulatory arterial stiffness index; PAIx: peripheral augmentation index; PWV: pulse wave velocity. $p$ values: (a): 0.166; (b): 0.469; (c): 0.076; (d): 0.825; (e): 0.102; (f): 0.090 .

After dividing the sample into quartiles according to SOD serum levels, the values of PAIx, AASI, PWV, and cardiovascular risk estimated with D'Agostino index are higher in the first quartile, although only AASI shows statistically significant differences ( $p=0.019$ ) (Table 3$)$. This trend was maintained after adjusting for age in the case of PAIx and AASI (Figure 1).

\section{Discussion}

This is the first study linking serum SOD levels with PWV, AIx, AASI, and pulse pressure, thus suggesting that oxidative stress significantly affects the vascular structure and function in hypertensive and diabetic patients and showing the role of SOD as an indicator of hypertension and diabetes-induced 
TABLE 3: Parameters of vascular structure and function divided into 4 quartiles according to superoxide dismutase serum levels.

\begin{tabular}{lcccc}
\hline & $\begin{array}{c}\text { Q1 } \\
\text { (SOD: }<87.3 \mathrm{ng} / \mathrm{mL})\end{array}$ & $\begin{array}{c}\text { Q2 } \\
(\mathrm{SOD}: 87.3-112.8 \mathrm{ng} / \mathrm{mL})\end{array}$ & $\begin{array}{c}\text { Q3 } \\
(\mathrm{SOD}: 112.8-142.6 \mathrm{ng} / \mathrm{mL})\end{array}$ & $\begin{array}{c}\text { Q4 } \\
(\mathrm{SOD}:>142.6 \mathrm{ng} / \mathrm{mL})\end{array}$ \\
\hline $\begin{array}{l}\text { IMT medium average } \\
(\mathrm{mm})\end{array}$ & $0.73 \pm 0.11$ & $0.75 \pm 0.13$ & $0.73 \pm 0.10$ & $0.72 \pm 0.12$ \\
$\begin{array}{l}\text { IMT maximum average } \\
(\mathrm{mm})\end{array}$ & $0.90 \pm 0.12$ & $0.92 \pm 0.16$ & $0.90 \pm 0.12$ & 0.436 \\
PWV (m/s) & $9.64 \pm 2.47$ & $8.83 \pm 1.94$ & $8.83 \pm 2.46$ & $0.89 \pm 0.14$ \\
Central AIx & $29.41 \pm 9.87$ & $30.72 \pm 9.50$ & $27.04 \pm 10.67$ & $8.88 \pm 1.81$ \\
Peripheral AIx & $91.92 \pm 22.84$ & $87.72 \pm 14.71$ & $85.32 \pm 22.48$ & 0.088 \\
AASI & $39.62 \pm 6.40$ & $39.29 \pm 6.69$ & $37.64 \pm 5.84$ & $84.05 \pm 22.82$ \\
$\begin{array}{l}\text { D'Agostino } \\
\text { cardiovascular risk }\end{array}$ & $21.75 \pm 19.30$ & $18.30 \pm 13.79$ & $19.92 \pm 17.11$ & $36.74 \pm 5.34$ \\
\hline
\end{tabular}

Data are expressed as mean \pm standard deviation. AASI: ambulatory arterial stiffness index; AIx: augmentation index; CV cardiovascular; IMT: intima-media thickness; $p$ : $p$ value, statistically significant differences (ANOVA); PWV: pulse wave velocity.

impairment of cardiovascular function, cardiovascular risk, and target organ damage. After adjusting for age, we still detect correlations between SOD and PAIx, AASI, and IMT, confirming the role of this serum marker as predictor of alterations in vascular structure and function.

Serum levels of SOD are negatively correlated with PWV, PAIX, CAIx, and AASI in our study population (hypertensive and diabetic patients recruited from primary care centers), thus suggesting that oxidative stress promotes or encourages the development of endothelial dysfunction, as it has been previously suggested by other authors [22]. Although the role of CAIx in the clinical setting remains unclear, it seems to be a predictor of adverse cardiovascular events in several patient populations, and higher AIx is associated with target organ damage [10]. According to our data, it has been previously observed that SOD improves endothelial function [23] and $\mathrm{Mn}-\mathrm{SOD}$ protects against oxidative stress and endothelial dysfunction in ApoE-deficient mice [24].

We detected negative correlations between serum SOD and most of the parameters of vascular structure and function analysed. Our data suggest that serum SOD is not increased in response to vascular injury in hypertensive and diabetics patients; its serum levels are not increased in these patients with vascular disorders, but its decline indicates a deficit in antioxidant defence mechanisms, since hypertensive and diabetic patients are unable to remove the circulating superoxide anion and therefore suffer an increase in vascular damage induced by reactive oxygen species. Thus, a lower level of serum SOD is associated with increased vascular damage.

We observed a negative correlation between SOD and HDL-cholesterol and positive correlation between SOD and triglycerides. HDL-cholesterol levels are inversely related to the risk of clinical events due to atherosclerosis [23]. On the other hand, a univariate association between triglycerides and cardiovascular risk has been described [25]. However, although the relationship between SOD serum levels with HDL-cholesterol and triglycerides has not been previously described, our results suggest that these molecules are related but further experiments are needed to assess the nature and characteristics of this relationship.
In our study population, there are positive correlations between SOD and uric acid. In agreement with our finding, Brand et al. [26] described a strong correlation between hyperuricemia and elevated cardiovascular risk in the general population, and it has been observed that serum uric acid correlates with extracellular SOD activity in patients with chronic heart failure [27]. As we said previously regarding HDL-cholesterol and triglycerides, additional experiments are needed to elucidate the pathophysiological significance of this correlation.

Limitations of our study are its cross-sectional design, which precludes longitudinal analysis between SOD, vascular structure, and function. We have analysed SOD expression level, but not its activity. Sampling of the study was performed consecutively, including hypertensive patients with a short course of hypertension, or with diabetes and hyperlipidemia, and many patients receiving drug therapy, which may modify blood pressure levels. However, the heterogeneity of the sample is similar to the distribution of the real population of short-course hypertensive patients with some risk factors and without previous cardiovascular disease.

\section{Conclusions}

We describe for the first time that SOD serum levels are correlated with alterations in vascular structure and function, being an indicator of cardiovascular alterations, cardiovascular risk, and target organ damage in hypertensive and diabetic patients, thus confirming that oxidative stress negatively contributes to the proper functioning of blood vessels. These encouraging data have to be followed by prospective studies to establish the relative strength of the prediction of cardiovascular risk and the appearance of target organ damage according to the SOD levels presented by the patient.

\section{Abbreviations}

AASI: Ambulatory arterial stiffness index ACEi: Angiotensin converting enzyme inhibitors

AIx: Augmentation index 


$\begin{array}{ll}\text { ARB: } & \text { Angiotensin receptor blockers } \\ \text { CV: } & \text { Cardiovascular } \\ \text { DIA: } & \text { Diabetic nonhypertensive patients } \\ \text { DIA + HYP: } & \text { Diabetic hypertensive patients } \\ \text { HbAlc: } & \text { Glycosylated haemoglobin } \\ \text { HDL: } & \text { Cholesterol associated with high density } \\ & \text { lipoproteins } \\ \text { HYP: } & \text { Hypertensive patients } \\ \text { IMT: } & \text { Intima-media thickness } \\ \text { LDL: } & \text { Cholesterol associated with low density } \\ & \text { lipoproteins } \\ \text { PWV: } & \text { Pulse wave velocity } \\ \text { SOD: } & \text { Superoxide dismutase. }\end{array}$

\section{Conflict of Interests}

The authors declare that there is no conflict of interests regarding the publication of this paper.

\section{Authors' Contribution}

Manuel A. Gómez-Marcos, Luis García-Ortiz, and Carlos Martínez-Salgado designed the study; Manuel A. GómezMarcos, Ana M. Blázquez-Medela, Luis Gamella-Pozuelo, and José I. Recio-Rodriguez performed the study and collected data; Ana M. Blázquez-Medela, José I. RecioRodriguez, Luis Gamella-Pozuelo, Luis García-Ortiz, and Carlos Martínez-Salgado analysed data; Luis García-Ortiz and Carlos Martínez-Salgado wrote the paper. Luis GarcíaOrtiz and Carlos Martínez-Salgado contributed equally to this paper.

\section{Acknowledgments}

This work was supported by grants from Instituto de Salud Carlos III (Ministerio de Sanidad y Consumo, PS09/01067, PI12/00959, and Retic RD06/0018, Rediapp), Junta de Castilla y León (Excellence Group GR100, IES095U14), and Obra Social Caja de Burgos (Founding for Clinical Research 2009, 2010). Ana M. Blázquez-Medela is supported by Fondo de Investigaciones Sanitarias (FIS) from the Institute Carlos III, Ministerio de Ciencia e Innovación. The authors thank the collaboration of the members of the Vaso-risk group: Luis García-Ortiz, Manuel A. Gómez-Marcos, José I. RecioRodriguez, Cristina Agudo-Conde, Maria C Patino-Alonso, Emiliano Rodriguez-Sanchez, Emilio Ramos-Delgado, Luis J González-Elena, Ángel García-García, Javier Parra-Sánchez, Isabel Sánchez Herrero, Carmela Rodríguez-Martín, Carmen Castaño-Sánchez, Ángela de Cabo-Laso, Benigna SánchezSalgado, Carlos Martínez-Salgado, and Ana M. BlázquezMedela.

\section{References}

[1] L. Ghiadoni, S. Taddei, and A. Virdis, "Hypertension and endothelial dysfunction: therapeutic approach," Current Vascular Pharmacology, vol. 10, no. 1, pp. 42-60, 2012.
[2] E. Standl, B. Balletshofer, B. Dahl et al., "Predictors of 10-year macrovascular and overall mortality in patients with NIDDM: the Munich General Practitioner Project," Diabetologia, vol. 39, no. 12, pp. 1540-1545, 1996.

[3] D. Versari, E. Daghini, A. Virdis, L. Ghiadoni, and S. Taddei, "Endothelial dysfunction as a target for prevention of cardiovascular disease," Diabetes Care, vol. 32, pp. S314-S321, 2009.

[4] M. A. Potenza, S. Gagliardi, C. Nacci, M. R. Carratu, and M. Montagnani, "Endothelial dysfunction in diabetes: from mechanisms to therapeutic targets," Current Medicinal Chemistry, vol. 16, no. 1, pp. 94-112, 2009.

[5] J. L. Cavalcante, J. A. C. Lima, A. Redheuil, and M. H. Al-Mallah, "Aortic stiffness: current understanding and future directions," Journal of the American College of Cardiology, vol. 57, no. 14, pp. 1511-1522, 2011.

[6] C.-C. Chen, K.-C. Hung, I.-C. Hsieh, and M.-S. Wen, "Association between peripheral vascular disease indexes and the numbers of vessels obstructed in patients with coronary artery disease," American Journal of the Medical Sciences, vol. 343, no. 1, pp. 52-55, 2012.

[7] M. Kikuya, J. A. Staessen, T. Ohkubo et al., "Ambulatory arterial stiffness index and 24-hour ambulatory pulse pressure as predictors of mortality in Ohasama, Japan," Stroke, vol. 38, no. 4, pp. 1161-1166, 2007.

[8] H. Triantafyllidi, S. Tzortzis, J. Lekakis et al., "Association of target organ damage with three arterial stiffness indexes according to blood pressure dipping status in untreated hypertensive patients," American Journal of Hypertension, vol. 23, no. 12, pp. 1265-1272, 2010.

[9] M. A. Gómez-Marcos, J. I. Recio-Rodríguez, M. C. PatinoAlonso et al., "Ambulatory arterial stiffness indices and target organ damage in hypertension," BMC Cardiovascular Disorders, vol. 12, article 1, 2012.

[10] M. Shimizu and K. Kario, "Role of the augmentation index in hypertension," Therapeutic Advances in Cardiovascular Disease, vol. 2, no. 1, pp. 25-35, 2008.

[11] N. S. Dhalla, R. M. Temsah, and T. Netticadan, "Role of oxidative stress in cardiovascular diseases," Journal of Hypertension, vol. 18, no. 6, pp. 655-673, 2000.

[12] M. T. Elnakish, H. H. Hassanain, P. M. Janssen, M. G. Angelos, and M. Khan, "Emerging role of oxidative stress in metabolic syndrome and cardiovascular diseases: important role of Rac/NADPH oxidase," Journal of Pathology, vol. 231, no. 3, pp. 290-300, 2013.

[13] F. Lacy, D. T. O’Connor, and G. W. Schmid-Schönbein, "Plasma hydrogen peroxide production in hypertensives and normotensive subjects at genetic risk of hypertension," Journal of Hypertension, vol. 16, no. 3, pp. 291-303, 1998.

[14] C. R. White, T. A. Brock, L.-Y. Chang et al., "Superoxide and peroxynitrite in atherosclerosis," Proceedings of the National Academy of Sciences of the United States of America, vol. 91, no. 3, pp. 1044-1048, 1994.

[15] M. K. Cathcart, A. K. McNally, D. W. Morel, and G. M. Chisolm III, "Superoxide anion participation in human monocytemediated oxidation of low-density lipoprotein and conversion of low-density lipoprotein to a cytotoxin," Journal of Immunology, vol. 142, no. 6, pp. 1963-1969, 1989.

[16] T. Fukai and M. Ushio-Fukai, "Superoxide dismutases: role in redox signaling, vascular function, and diseases," Antioxidants and Redox Signaling, vol. 15, no. 6, pp. 1583-1606, 2011.

[17] G. Mancia, R. Fagard, K. Narkiewicz et al., "2013 ESH/ESC Guidelines for the management of arterial hypertension: the 
Task Force for the management of arterial hypertension of the European Society of Hypertension (ESH) and of the European Society of Cardiology (ESC)," Journal of Hypertension, vol. 31, pp. 1281-1357, 2013.

[18] American Diabetes Association, "Diagnosis and classification of diabetes mellitus," Diabetes Care, vol. 37, supplement 1, pp. S81-S90, 2014.

[19] E. O’Brien, R. Asmar, L. Beilin et al., "Practice guidelines of the European Society of Hypertension for clinic, ambulatory and self blood pressure measurement," Journal of Hypertension, vol. 23, no. 4, pp. 697-701, 2005.

[20] M. A. Gómez-Marcos, J. I. Recio-Rodríguez, M. C. PatinoAlonso et al., "Protocol for measuring carotid intima-media thickness that best correlates with cardiovascular risk and target organ damage," American Journal of Hypertension, vol. 25, no. 9, pp. 955-961, 2012.

[21] R. B. D’Agostino Sr., R. S. Vasan, M. J. Pencina et al., "General cardiovascular risk profile for use in primary care: the Framingham heart study," Circulation, vol. 117, no. 6, pp. 743-753, 2008.

[22] J. H. Indik, S. Goldman, and M. A. Gaballa, "Oxidative stress contributes to vascular endothelial dysfunction in heart failure," The American Journal of Physiology-Heart and Circulatory Physiology, vol. 281, no. 4, pp. H1767-H1770, 2001.

[23] M. Navab, G. M. Anantharamaiah, S. T. Reddy, B. J. Van Lenten, B. J. Ansell, and A. M. Fogelman, "Mechanisms of disease: proatherogenic HDL-an evolving field," Nature Clinical Practice Endocrinology \& Metabolism, vol. 2, no. 9, pp. 504-511, 2006.

[24] M. Ohashi, M. S. Runge, F. M. Faraci, and D. D. Heistad, "MnSOD deficiency increases endothelial dysfunction in ApoE-deficient mice," Arteriosclerosis, Thrombosis, and Vascular Biology, vol. 26, no. 10, pp. 2331-2336, 2006.

[25] M. A. Austin, B. L. Rodriguez, B. McKnight et al., "Lowdensity lipoprotein particle size, triglycerides, and high-density lipoprotein cholesterol as risk factors for coronary heart disease in older Japanese-American men," The American Journal of Cardiology, vol. 86, no. 4, pp. 412-416, 2000.

[26] F. N. Brand, D. L. McGee, W. B. Kannel, J. Stokes III, and W. P. Castelli, "Hyperuricemia as a risk factor of coronary heart disease: the Framingham study," The American Journal of Epidemiology, vol. 121, no. 1, pp. 11-18, 1985.

[27] H. Alcaino, D. Greig, M. Chiong et al., "Serum uric acid correlates with extracellular superoxide dismutase activity in patients with chronic heart failure," European Journal of Heart Failure, vol. 10, no. 7, pp. 646-651, 2008. 


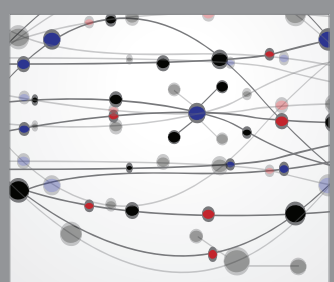

The Scientific World Journal
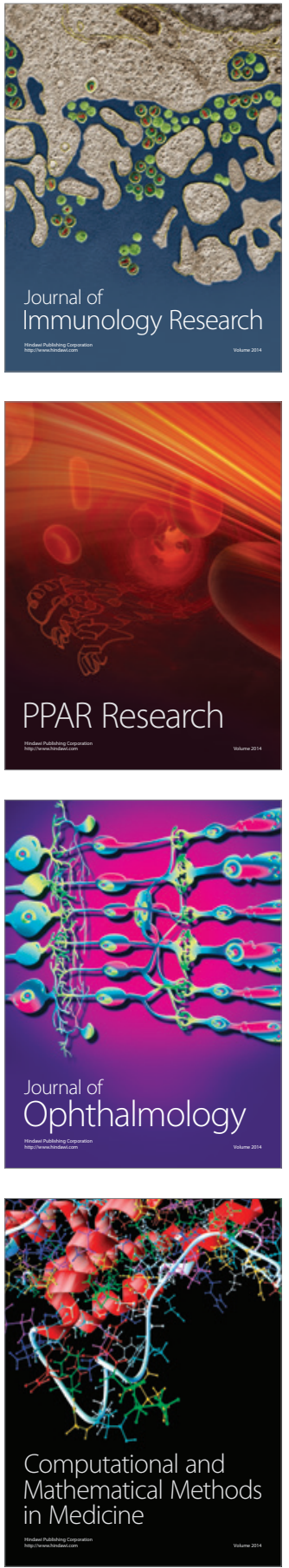

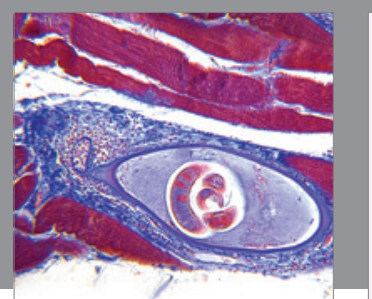

Gastroenterology Research and Practice

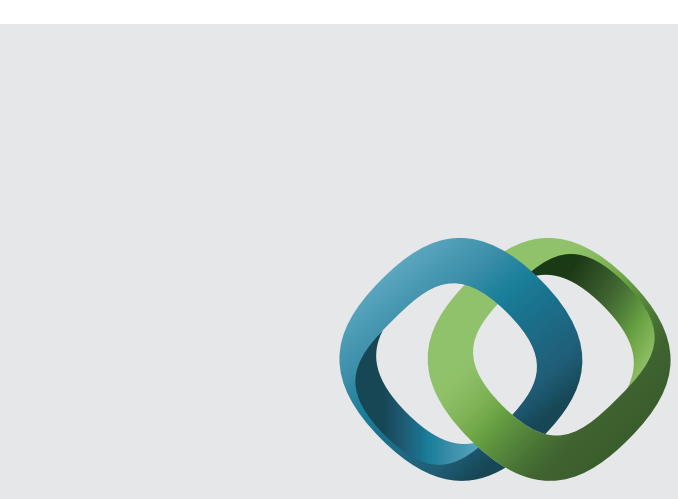

\section{Hindawi}

Submit your manuscripts at

http://www.hindawi.com
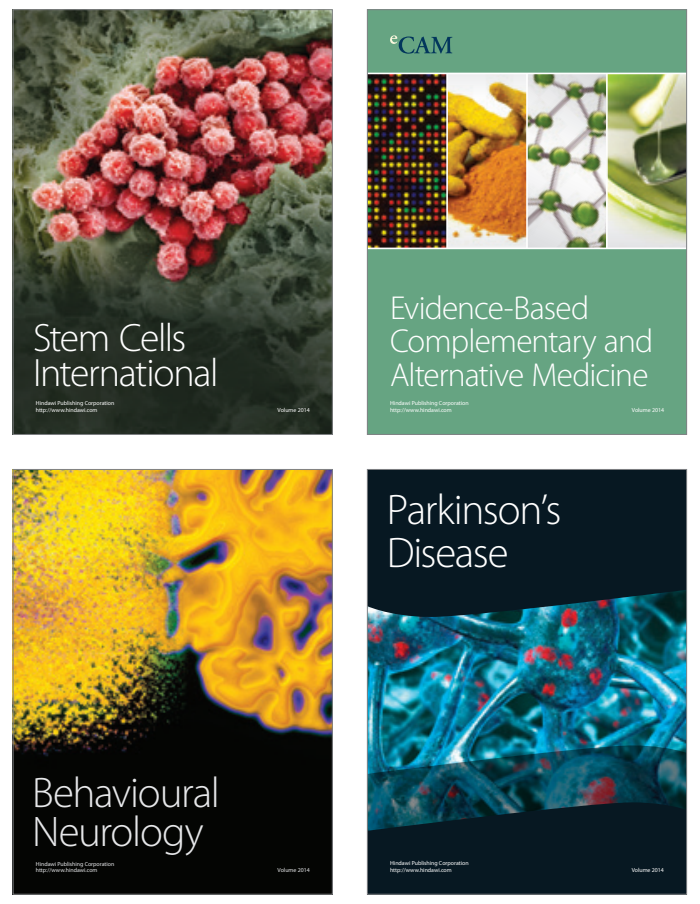
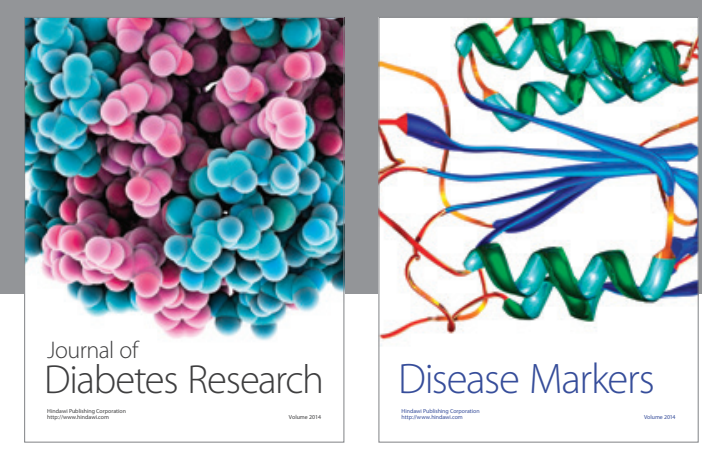

Disease Markers
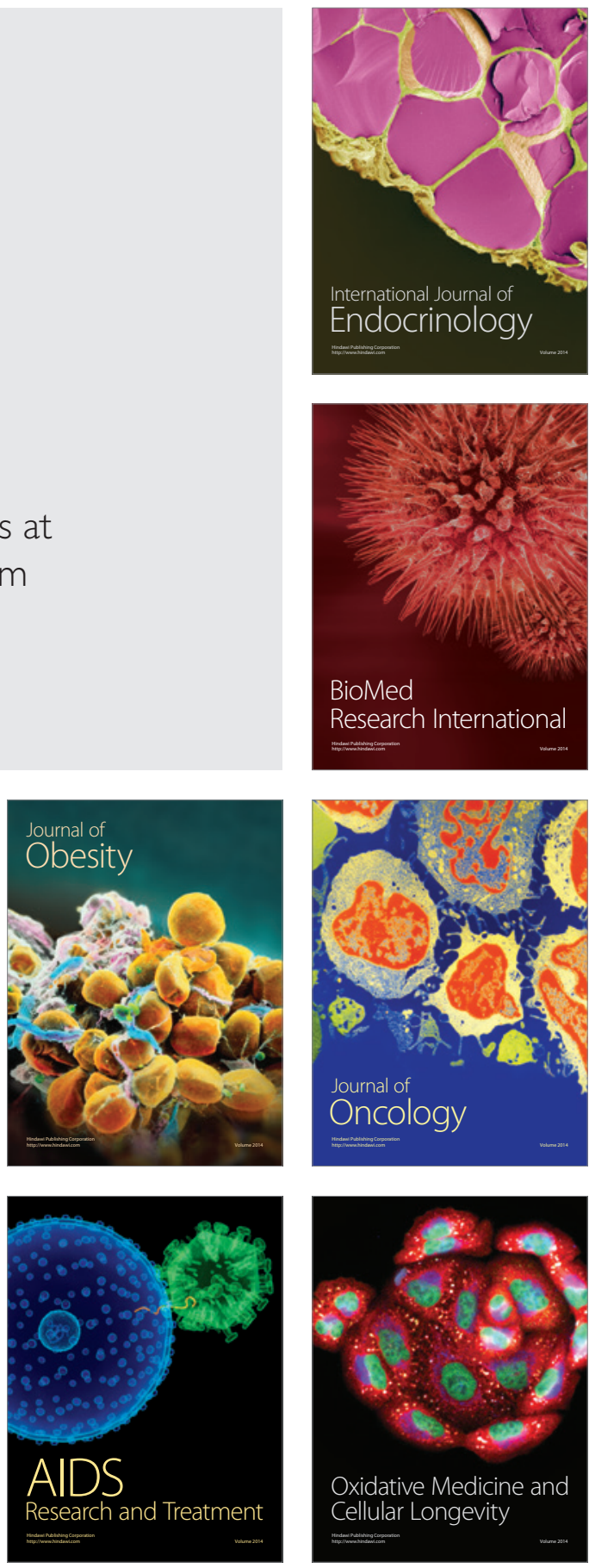